\title{
Full Reference Image Quality Assessment of Perceptual Distortion based on Image Retargeting
}

\author{
S. Shigwan and G. Birajdar \\ Dept. of Electronics \& Communication, Pillai HOC CoE \&T, Raigad, M.S., India. 410206 \\ \{shigwan.supriya@gmail.com,gajanan123@gmail.com\}
}

\begin{abstract}
Image quality assessment is used for automatic evaluation of the image accurately. In this article, objective reference image quality assessment metric is used to evaluate the quality of retargeted images. The metric measures perceptual geometric distortion and information loss of retargeted images using deformable spatial pyramid (DSP) algorithm. NRID and RetargetMe database are employed to measure the performance of the metric. Experimental results shows that the performance of DSP algorithm is superior thanScale-invariant feature transform (SIFT) in terms of runtime and accuracy. Image retargeting, fast dense correspondence, Image quality assessment, deformable spatial pyramid, full reference image quality assessment
\end{abstract}

\section{Introduction}

Nowadays, there is an increase demand in mobile devices like cellular phones and personal digital assistants. The images have high resolutions and various aspect ratios than the small screens of the mobile devices. To fit random size images of various aspect ratios, the image retargeting technique reduces/expands the images by automatically removing/adding less-important image plots thereby keeping the important features intact. This property is useful to create images of different sizes, which is adapted for various display devices like mobile, TV and personal digital assistants, etc. that usually have widely different resolutions. The common result to image retargeting is to distinctively rescale the source image according to the output screen size. Image quality is a characteristic of an image that estimates the image degradation of test image with a reference image. During various processing stages, digital images are subject to various types of distortions which degrade the visual quality of image.

In this paper, in order to demonstrate the perceptual quality of retargeted image, a full reference image quality metric is presented. The two major distortions, the geometric distortion and information loss are observed to evaluate quality of retargeted images. These distortions are evaluated by using DSP algorithm. The contributions are

- Estimation of the perceptual distortion, information loss distortion metric and fusion of both distortion metric of retargeting images is demonstrated in [3] using SIFT algorithm. To overcome the limitations like low accuracy and high execution time, DSP algorithm is used in place of SIFT.

- We show that, the performance of DSP algorithm is more effective than the SIFT algorithm.

- We analyze the rank correlation and error values using DSP algorithm for various retargeted schemes using NRID[16] and RetargetMe[15] database.

In Section 2, an overview of the related work is discussed. In section 3, the system model is presented. In 4,5 and 6 sections the perceptual geometric distortion and saliency loss ratio metrics and the final quality metric which consists of fusion of two metrics is explained. In section 7 experimental results are represented and Section 8 concludes an article.

\section{Related Work}

B. Iyer, S. Nalbalwar and R. Pawade (Eds.)

ICCASP/ICMMD-2016. Advances in Intelligent Systems Research.

Vol.137, Pp. 404-411.

(C) 2017- The authors. Published by Atlantis Press

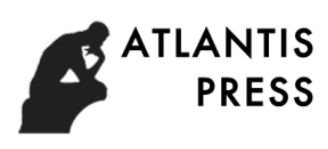

This is an open access article under the CC BY-NC license (http://creativecommons.org/licens)es/by-nc/4.) 
Image quality assessment is classified into objective and subjective quality assessment. Subjective image quality assessment methods are very expensive and time consuming and hence difficult to perform in real-time applications. Therefore, it can be done objectively using computer algorithms or statistical models. The main focus of objective image quality metric is to design mathematical models which predict the accurate image quality of an image automatically.

The classification of objective image quality assessment is based on availability of original image. Image quality assessment (IQA) algorithm can be classified as full reference IQA, reduced reference IQA and no reference IQA. Full reference IQA means that the reference image is fully available, reduced reference IQA means that the information about reference image is partially available and no reference IQA refers to the image quality assessment without a reference image. The regular metrics are mean squared error (MSE) and peak signal to noise ratio (PSNR). These methods are simple but fail to evaluate the visual quality accurately.

The main objectives of image retargeting are 1) to preserve the important content of the source media 2) to limit visual artifacts in the resulting media and 3) to preserve internal structures of the source media. Conventional retargeting methods such as scaling, shifting and cropping affected by various distortions or loss of important information [10][1][13]. To overcome this problem, various algorithm for content-aware image retargeting have been proposed to preserve important image information during the image resizing process [5][9][11][6]. One of the popular image retargeting algorithms is seam carving [10], which reducesthe width or height of an image by removing the unimportant seams. The seam carving algorithm is also used for video retargeting [11][6].

Wang et al. [13] proposed an image retargeting algorithm with a combination of both saliency plot and a gradient plot. Wolf et al. [5] presented a linear system for designing an image retargeting algorithm. In [3], SIFT algorithm is used to compute the perceptual distortions of retargeted images. But performance of this algorithm is poor in terms of accuracy and execution time. To get the best of these limitations DSP algorithm is used.

\section{System Model}

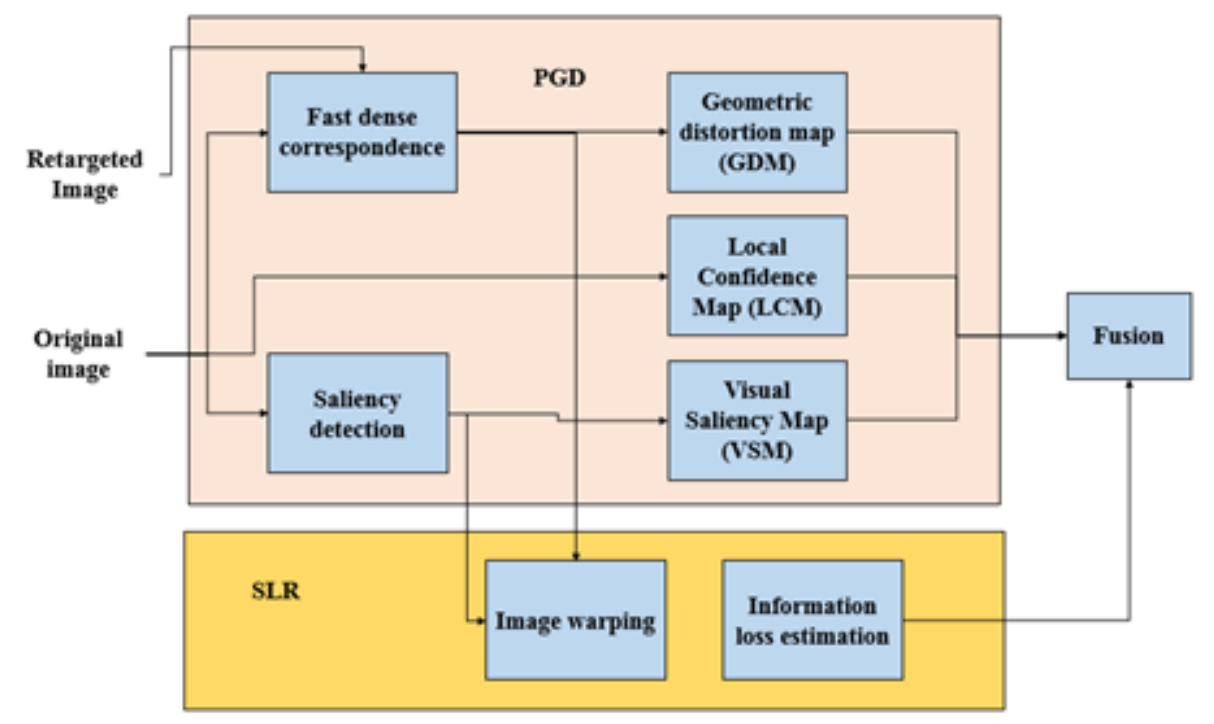

Fig. 1: Architecture of image quality assessment using DSP algorithm

Fig 1 shows the architecture of image quality assessment using DSP algorithm which is derived from [3]. The perceptual geometric distortion (PGD) is used to measure a pixel wise dense correspondence plot. It is calculated by comparing the original image and its retargeted image. The fast dense corresponding scheme is used to evaluate the correspondence plot between the source and the retargeted images. A visual saliency plot is calculated by estimating the weights of plot wise geometric distortions using the important visual information of the plots and then a local confidence map (LCM) is evaluated.

The metric for calculating the loss of important data which create distortion in image is known as the information loss which denotes the proportion of the additions of saliency values of the images after applying retargeting and before applying retargeting. To get the retargeted image, warp the saliency plot ofsource image based on the pixel 
given by the DSP plot. The pixel-wise saliencyvalues in the retargeted saliency plot are combined to obtain the saliency loss ratio metric.

\section{Perceptual geometric distortion}

Geometric distortion can happen due to the direct application of geometric transformations. To calculate geometric distortion three plots are required: a) Geometric distortion, b) visual saliency and c) local confidence. The energy function is computed to find the optimal translations as:

$$
E(\mathrm{t})=\sum_{i} H_{i}\left(r_{i}\right)+\alpha \sum_{i, j \in G} X_{i, j}\left(r_{i}, r_{j}\right)
$$

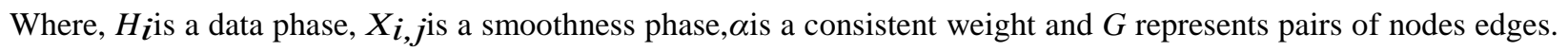
The $H_{\boldsymbol{i}}$ is used for measurement of matching cost and is given as:

$$
H_{i}\left(r_{i}\right)=\frac{1}{y} \sum_{i} \min \left(\| P_{1}(t)-P_{2}\left(t+\mathrm{b}_{i} \| 1\right), \lambda\right)
$$

Where, $t$ represents the pixel coordinates.

For measurement of the local geometric distortion in a retargeted image, GDM is used. Let us assume that the source image $\mathrm{K}_{0}$ is resized from $\mathrm{b}_{0} \times \mathrm{d}_{0}$ to $\mathrm{b}_{\mathbf{r}} \times \mathrm{d}_{\mathbf{r}}$. Let $\mathrm{v}\left(\mathrm{K}_{\mathbf{i}}\right)$ and $\mathrm{u}\left(\mathrm{K}_{\mathbf{i}}\right)$ denote the horizontal and vertical component vector of patch $\mathrm{K}_{\mathbf{i}}$. The GDM can be calculated by the following equation,

$\boldsymbol{q}_{G D M}(\mathrm{Ki})=\frac{\mathrm{rB}}{\mathbf{r B}+\mathrm{rD}} \operatorname{var}(\mathrm{u}(\mathrm{Ki}))+\frac{\mathrm{rD}}{\mathbf{r B}+\mathbf{r D}} \operatorname{var}(\mathrm{v}(\mathrm{Ki}))$

Where $r_{B}=B_{r} / B_{0}$ and $r_{D}=D_{r} / D_{0}$ represent the ratio of the width and height of the retargeted image to that of the source image $\mathrm{K}_{0}$, where $0<\mathrm{r}_{\mathrm{B}}, \mathrm{r}_{\mathrm{D}}<1$, and $\operatorname{var}($.) denotes the variance function.

\subsection{Local confidence map (LCM)}

The LCM of a patch is estimated as:

$q_{L C M}(\mathrm{Ki})=\frac{1}{\mathrm{Pk}} \sum_{k \in k i} \frac{1}{\max (|\operatorname{Do}(k)-\operatorname{Dr}(k+w(k))|, \epsilon)}$

Where $\mathrm{P}_{\mathrm{k}}$ represents the plot size, $\mathrm{w}(\mathrm{k})$ denotes the SIFT flow vector of pixel $\mathrm{k}, \mathrm{k}+\mathrm{w}(\mathrm{s})$ is the corresponding pixel location after the compensation, $\operatorname{Dr}(\mathrm{k}+\mathrm{w}(\mathrm{k}))$ is the de-warped image based on $\mathrm{w}$.

The VSM of a patch is estimated as follows:

$q_{V S M}\left(\mathrm{~K}_{\mathrm{i}}\right)=\frac{1}{P_{k}} \sum_{k \in k i} E o(k)$

Where, $P_{k}$ represents the patch size.

The final perceptual geometric distortion of a retargeted image is calculated as follow:

$Z_{P G D=\frac{1}{Q_{n}} \sum_{i=1}^{Q n} q_{G D M}\left(k_{i}\right) q_{L C M}(K i) q_{V S M}\left(K_{i}\right)}$

Where, $Q_{n}$ represents the number of patches in the fast dense correspondence plot.

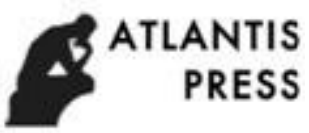




\section{Information loss}

Measurement of the information loss of image retargeting is done by SLR metric. Let $I_{0}$ and $I_{r}$ represent the saliency slots of the source and retargeted images respectively. The SLR metric is computed by obtaining the ratio of the saliency amount of trimming in the retargeted image and the source image. We use DSP algorithm to obtain the retargeted saliency map as follows:

$\mathrm{I}_{\mathbf{r}}(\mathrm{k})=\mathrm{I}_{0}(\mathrm{k}+\mathrm{w}(\mathrm{t}))$

Where, $I_{r}(s)$ represents the warped pixel-wise saliency plot. As a result, the size of $I_{r}$ is the same as that of the test image. The SLR is estimated as follows:

$Z_{S L R}=1-\frac{\sum I_{r}(s)}{\sum I_{O}(s)}$

The SLR value is in the range of $[0,1]$.

\section{Combination of PGD and SLR}

The retargeting image quality factor $T_{\text {resize }}$ is obtained by subtraction of the overall distortion $Z_{\text {resize }}$ from unity:

Tresize $=1-Z_{\text {resize }}=1-\alpha . Z_{S L R}+(1-\alpha) \cdot Z_{P G D}$

Where, $z_{\text {resize }}$ is summation of the PGD and SLR indices within the range of $[0,1]$ and to control weights $\alpha$ is used.

\section{Experimental results}

In this experiment, the accuracy of the full reference image quality metric by using the dataset RetargetMe dataset [8] database and NRID database [7] is evaluated. The sample images from RetargetMe database are shown in Fig 3. For this dataset we have compared all the eight retargeted schemes used in [8]. SIFT descriptors of $16 \times 16$ plot size at every pixel using VLFeat [12] are extracted. The experiments are performed using Matlab running on core i3 processor. To estimate the saliency plot, ground truth method is employed. The 37 images in the RetargetMe dataset are classified into the following attributes: line, face, texture, geometric structure, symmetry and foreground objects. One image may contain multiple attributes as shown in Fig 4. from RetargetMe dataset [8].

The Kendall distance [4] is calculated as follows:

$$
\Gamma=1-\frac{M_{c}-M_{d}}{0.5 M(\mathrm{M}-1)}
$$

Where, $M$ is the aggregate number of pairs for comparison and $M_{d}$ and $M_{c}$ represents the numbers of discordant pairs and concordant pairs respectively.

Table 1: Comparison of Rank Correlation Values for NRID database

\begin{tabular}{|c|c|c|c|c|c|c|c|c|}
\hline \multirow[b]{2}{*}{ Metric } & \multicolumn{6}{|c|}{ Attributes } & \multirow[b]{2}{*}{ Mean } & \multirow[b]{2}{*}{ std } \\
\hline & Lines & Faces & Textur & $\begin{array}{l}\text { Foreground } \\
\text { object }\end{array}$ & $\begin{array}{l}\text { Geometric } \\
\text { Structure }\end{array}$ & Symmetry & & \\
\hline DSP & 0.208 & 0.34 & 0.2678 & 0.252 & 0.225 & 0.33 & 0.249 & 0.2489 \\
\hline SIFT & 0.154 & 0.0952 & 0.017 & 0.025 & 0.1204 & 0.2738 & 0.138 & 0.321 \\
\hline
\end{tabular}




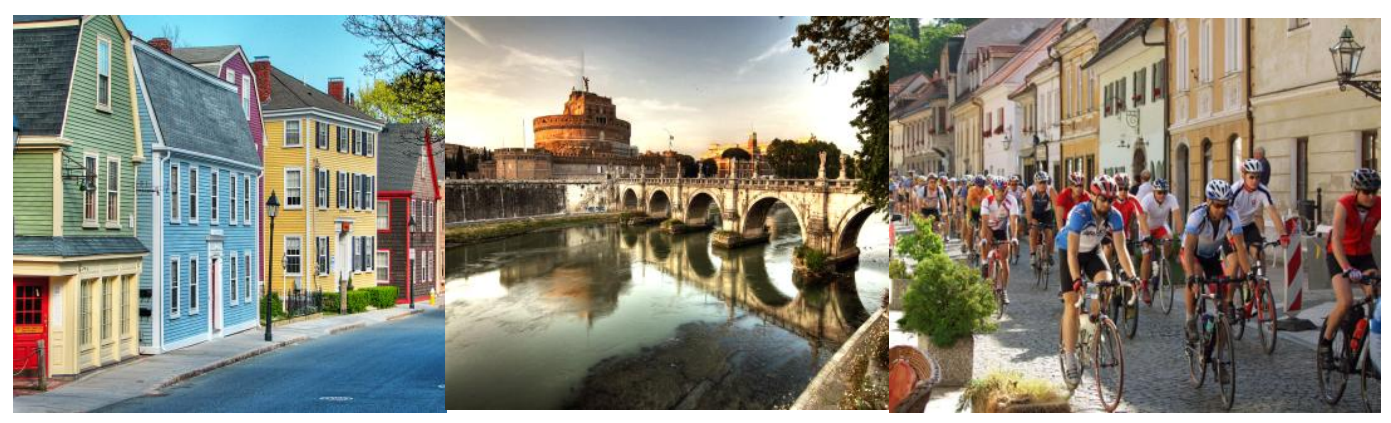

Fig. 2: Three example images from the RetargetMe dataset

Table2: Comparison of Rank Correlation Values for Retarget Medatabase

\begin{tabular}{|c|c|c|c|c|c|c|c|c|}
\hline \multirow[b]{2}{*}{ Metric } & \multicolumn{6}{|c|}{ Attribute } & \multirow[b]{2}{*}{ Mean } & \multirow[b]{2}{*}{ Std } \\
\hline & Lines & Faces & Texture & Foreground & $\begin{array}{l}\text { Geometric } \\
\text { Structure }\end{array}$ & Symmetry & & \\
\hline DSP & 0.093 & 0.6 & 0.41 & 0.0375 & 0.425 & 0.6 & 0.42 & 0.24 \\
\hline SIFT & 0.097 & 0.252 & 0.119 & 0.218 & 0.085 & 0.071 & 0.145 & 0.262 \\
\hline
\end{tabular}

Table 1 and 2 compares the rank correlation mean and standard deviation values for the DSP and SIFT metrics. The mean value of DSP is higher than that of SIFT. Higher the value better is the performance. It shows DSP that the algorithm is accurate than the SIFT algorithm. The rank correlation value for the lines/edges attribute images is the lowest.

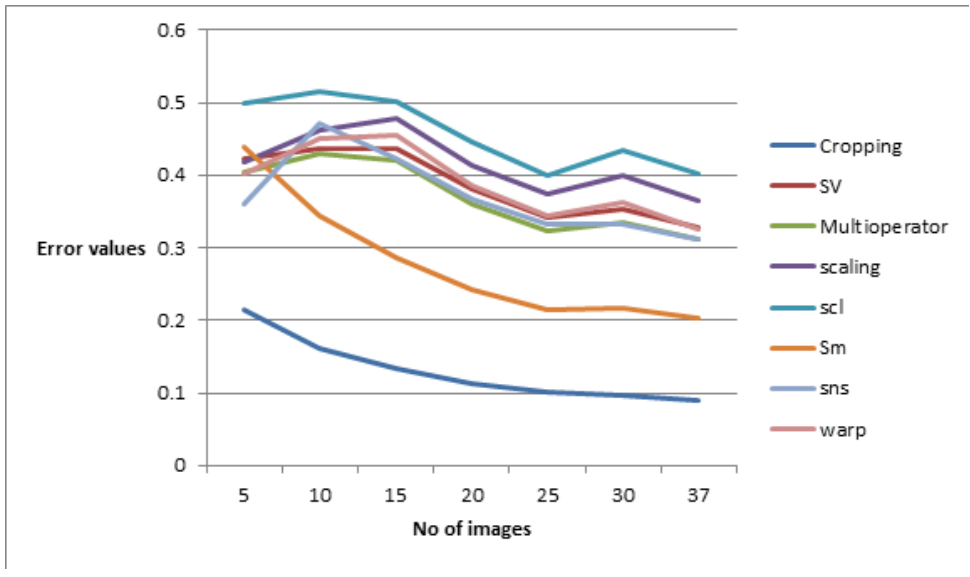

Fig. 3: Error values of various retargeted operators using DSP algorithm using RetargetMe database

It can be observed from Fig 3 and 4 that in most of the operators, the error values of DSP are lower compared to SIFT. If error value increases, then distortion in image increases. For example, in case of warping, the error value for DSP algorithm is less (0.2) whereas using SIFT it is multi-operator (0.4). Similarly, scaling image has high error value in DSP (0.0914) and in SIFT algorithm SM. Lower error values indicates that better performance of DSP algorithm compared to SIFT in terms of accuracy of image quality metric. It is also observed from Fig 5 and 6 that in most of the operators, the error values of DSP are lower compared to SIFT. When error value increases, distortion in image increases. For example, in case of warping, the error value for DSP algorithm is less (0.03) whereas using SIFT retargeted scheme, it is shift (0.004). 


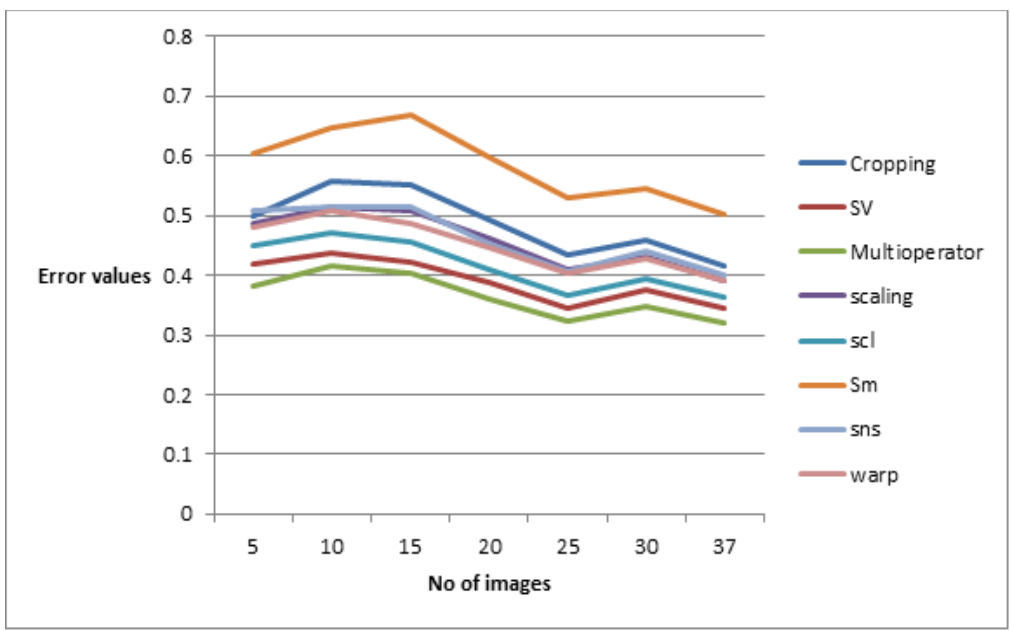

Fig. 4: Error values of various retargeted operators using SIFT algorithm using RetargetMe database



Fig. 5: Error values of various retargeted operators using DSP algorithm using NRID database

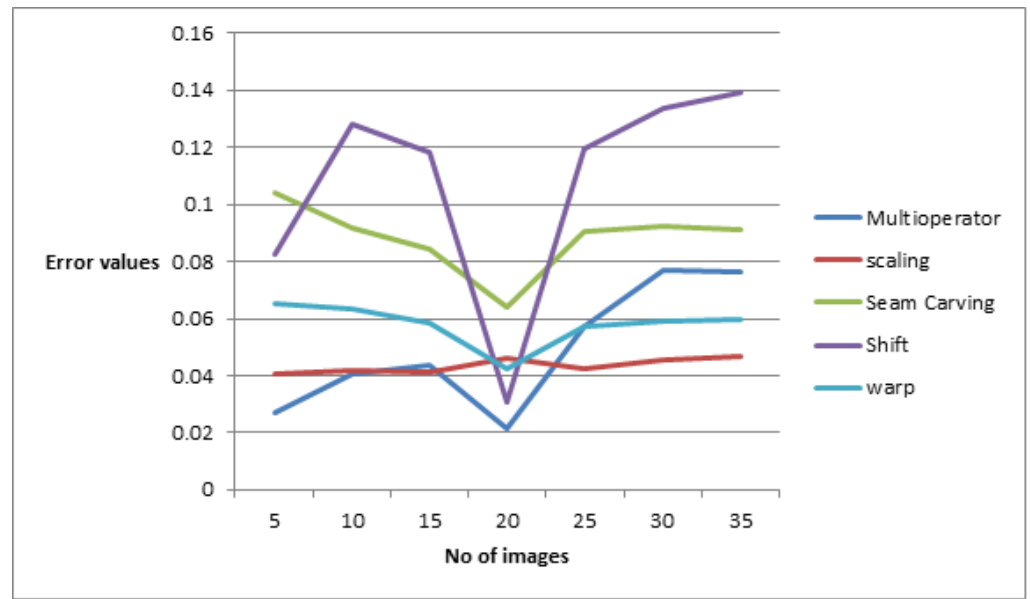

Fig. 6: Error values of various retargeted operators using SIFT algorithm using NRID database 
Similarly, seam carving image has high error value in DSP (0.1) and in SIFT algorithm retargeted scheme, it is multioperator (0.22). Lower error values indicate better performance of DSP algorithm compared to SIFT in terms of accuracy of image quality metric.

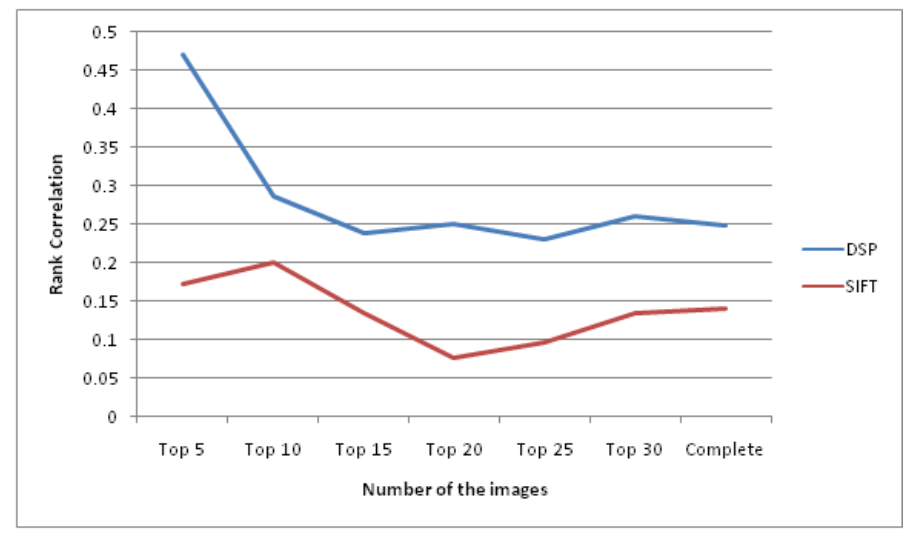

Fig. 7: Comparison of rank correlation values

Fig 7 shows the comparison of rank correlation values between subjective and objective paired ranking using two objective quality metrics for images in RetargetMe database. It shows that DSP algorithm has high correlation values than SIFT resulting in high accuracy.

Table 3: Time requirement of experiment performed by DSP and SIFT algorithm using RetargetMe database

\begin{tabular}{l} 
Algorithm Time $(\mathrm{min})$ \\
DSP(Proposed)35min33sec \\
SIFT(Original) $\quad 60 \mathrm{~min}$ \\
\hline
\end{tabular}

In table 3, the execution time required to perform the experiment using two algorithms are shown. The time required for DSP and SIFT is $35 \mathrm{~min} 33 \mathrm{sec}$ and 60 min respectively. Compared to SIFT flow algorithm, DSP algorithm require less execution time.

\section{Conclusion}

In this paper, objective image quality assessment of retargeted images using DSP algorithm is presented. The perceptual geometric distortion metric and information distortion metric and fusion of both metrics is analyzed using NRID and RetargetMe database. An experimental result shows that the DSP algorithm is better than SIFT algorithm because of its higher accuracy. This method is faster than SIFT in terms of execution time. The presented system can be further implemented using [2] method under various camera lens, various background and under various lighting.

\section{References}

[1] Guo, Y., Liu, F., Shi, J., Zhou, Z.H., M, G.: Image retargeting using mesh parameterization. IEEE Transactions on Multimedia11(5), 856-867(May 2009).

[2] HaCohen,Y.,Shechtman,E.,Goldman,D.B., Lischinski, D.:Non-rigid dense correspondence with applications for image enhancement. ACM Trans.Graph.30(4),70:1-70:10 (Jul 2011), http://doi.acm.org/10.1145/2010324.1964965.

[3] Hsu,C.C., Lin, C.W., Fang, Y.,Lin, W.: Objective quality assessment for image retargeting based on perceptual geometric distortion and information loss. IEEE Journal of Selected Topics in Signal Processing: 8 (3), 377-389(June2014).

[4] Kendall,M..:A new measure of rank correlation. Biometrika 30(1/2),81-93(1938). 
[5] 5.L.,W.,M.,G.,D,C.O.:Non-homogeneous content-driven video- retargeting. In:IEEE International Conference on Computer Vision. Pp.1-6(October2007).

[6] Li,B.,Duan, L.Y.,Wang, J.,Ji,R.,Lin,C.W., Gao,W.:Spatio-temporal Grid Flow for Video Retargeting. IEEE Transactions on Image Processing, 23, 1615-1628, Apr.2014.

[7] Ma, L.,Lin, W., Deng, C., Ngan, K.N.: Image retargeting quality assessment :A study of subjective scores and objective metrics. IEEE Journal of Selected Topics in Signal Processing, 6 (6), 626-639 (2012).

[8] Rubinstein, M., Gutierrez, D., Sorkine, O., Shamir, A.: A comparative study of image retargeting. In: ACM SIGGRAPH A s i 2010 Papers. pp.160:1-160:10. SIGGRAPH ASIA'10, ACM, New York, NY, USA (2010), http://doi.acm.org/10.1145/1866158.1866186.

[9] Rubinstein, M.,Shamir, A., Avidan,S.:Multi-operator media retargeting .ACM Transactions on Graphics (TOG)28(3)(2009).

[10] Shai, A.,Ariel, S.: Seam carving for content-aware image resizing. ACM Transactions on Graphics, 26 (3) (July 2007).

[11] Tzu-ChiehYen,Chia-MingTsai,Chia-Wen Lin: Maintaining Temporal Coherence in Video Retargeting Using Mosaic-Guided Scaling, IEEE TransactionsonImageProcessing20,2339-2351( Aug 2011).

[12] Vedaldi, A., Fulkerson,B.: VLFeat: An openand p ortable library of computer vision algorithms .http://www.vlfeat.org/(2008).

[13] Wang, Y.S., Tai, C.L., Sorkine, O., Lee, and T.Y.: Optimized scale-and-stretch forimage resizing. In: SIGGRAPH Asia 2008(2008). 